\title{
Quantum e-commerce: A comparative study of possible protocols for online shopping and other tasks related to e-commerce
}

\author{
Kishore Thapliyal*, Anirban Pathak ${ }^{\dagger}$ \\ Jaypee Institute of Information Technology, A-10, Sector-62, Noida, UP-201307, India
}

\begin{abstract}
A set of quantum protocols for online shopping is proposed and analyzed to establish that it is possible to perform secure online shopping using different types of quantum resources. Specifically, a single photon based, a Bell state based and two 3-qubit entangled state based quantum online shopping schemes are proposed. The Bell state based scheme, being a completely orthogonal state based protocol, is fundamentally different from the earlier proposed schemes which were based on conjugate coding. One of the 3-qubit entangled state based scheme is build on the principle of entanglement swapping which enables us to accomplish the task without transmission of the message encoded qubits through the channel. Possible ways of generalizing the entangled state based schemes proposed here to the schemes which use multiqubit entangled states is also discussed. Further, all the proposed protocols are shown to be free from the limitations of the recently proposed protocol of Huang et al. (Quantum Inf. Process. 14, 2211-2225, 2015) which allows the buyer (Alice) to change her order at a later time (after initially placing the order and getting it authenticated by the controller). The proposed schemes are also compared with the existing schemes using qubit efficiency.
\end{abstract}

\section{Introduction}

In today's society e-commerce plays a crucial role. Especially, we often purchase things from online stores. Such a purchase requires online transaction, and that requires security measures. So far our online transactions are secured by classical protocols, but it is well established that the majority of the classical security measures are vulnerable and they will not remain useful in a post-quantum world (once a scalable quantum computer is built) [1]. Thus, we need quantum protocols for e-commerce. This is so because security of any classical cryptographic protocol is based on some assumptions on the computational power of Eve. In contrast, quantum cryptographic protocols are unconditionally secure. This fact is known since the introduction of first quantum key distribution (QKD) protocol in 1984 [2]. Since then several quantum protocols have been proposed for various practical tasks that require security ( [2-18] and references therein). For example, a set of schemes for QKD [2-6], direct secure quantum communication [7--15], and its controlled counterpart-controlled deterministic secure quantum communication (CDSQC) [16, 17], have been proposed in the recent past (see [18] for a review). A recent addition to this long list is quantum e-commerce [19-24]. Specifically, in 2014, a three-party quantum protocol for online shopping was proposed by Chou et al. [23]. In this protocol, Alice is a buyer, Bob is a merchant (say a representative of Walmart, Big Bazaar or any other departmental stores which sales goods through e-commerce corporation like eBay, Flipkart or Amazon) and Charlie is a controller who may be considered as a representative of VISA or Master card or a representative of an e-commerce corporation like, eBay, Flipkart or Amazon. Chou et al.'s scheme allows Alice to buy a product from Bob in a secure manner. However, in Chou et al.'s protocol Charlie can obtain the information encoded by Alice (i.e., which product she wishes to buy). This limitation of Chou et al.'s protocol [23] was noted by Huang et al. [24], and in 2015, they proposed an improved protocol for quantum online shopping which is free from the limitation of Chou et al.'s scheme. More recently, a semiquantum scheme for quantum online shopping has also been proposed by us [25]. Prior to these relatively new protocols, a set of protocols were introduced for quantum online shopping [19-22]. Specifically, e-payment systems were introduced using quantum group signature [19], quantum blind and group signature [20], quantum proxy blind signature [21], etc., and they were critically analyzed. For example, cryptanalysis of inter-bank e-payment protocol introduced in Ref. [21] was performed in [22]. Further, schemes based on blind signature have also been designed using quantum teleportation [26, 27]. It may be noted that Chou et al.'s quantum-communication-based protocol [23] and its improved version proposed by Huang et al. [24] are free from the limitations of early protocols [19-22] of quantum online shopping. Thus, we would concentrate on the possible improvement of Chou et al.'s protocol [23] and Huang et al.'s protocol [24]. Further, we aim to provide a large number of alternative paths

\footnotetext{
*tkishore36@yahoo.com

†anirban.pathak@gmail.com
} 
that may be used to realize quantum online shopping. These alternatives would provide choices to the experimentalists interested in implementing schemes of quantum online shopping, based on the quantum resources available with them and the noise present in the channel.

In what follows, we will show that the above mentioned schemes of quantum online shopping [19-24] are essentially modified schemes of CDSQC, thus, the protocols of CDSQC that are introduced in the recent papers of some of the present authors [17,25] can be modified suitably to develop quantum protocols for online shopping. Further, it will be shown that in Huang et al.'s protocol the buyer (Alice) can change her order at a later time (after initially placing the order and getting it authenticated by the controller). This undesirable feature can be removed by using our schemes of CDSQC-based online shopping.

The remaining part of the paper is organized as follows. In Section 2, we briefly introduce the existing schemes of quantum online shopping. Thereafter, in Section 3 , we propose four new protocols for quantum e-commerce and a few other alternatives to perform the task. Subsequently, in the same section, we also describe a set of other cryptographic tasks related to e-commerce which can be realized by slightly modifying the protocols proposed here. We further discuss the security and qubit efficiency of the proposed schemes in Sections 4 and 5 respectively. Finally, we conclude the paper in Section 6 .

\section{Existing protocols for quantum online shopping}

Before we briefly describe the existing protocols of quantum online shopping it will be apt to note a few background information. To begin with let us note that the security of all the existing protocols of secure quantum communication has been achieved in two alternative ways which may be referred to as BB84 subroutine and GV subroutine. Specifically, an additional set of $n$ qubits are prepared as verification qubits and inserted randomly in the set of $n$ travel qubits. The verification qubits are referred to as decoy qubits and they are used later to check whether any eavesdropping attempt has been made. The presence of Eve could be inferred from the error rate computed on these verification qubits. The two alternative subroutines differ in the types of decoy qubits used and the principle of security in each case. Specifically, in BB84 subroutine, the security comes in a manner analogous to the BB84 QKD protocol [2]. Specifically, it comes from non-commutativity and nocloning theorem, where the decoy qubits are prepared randomly in $\{|0\rangle,|1\rangle\}$ or $\{|+\rangle,|-\rangle\}$ basis. The sender and receiver compute the error rates from the qubits prepared by the sender and measured by the receiver in the same basis as any eavesdropping attempt would have led to mismatch. In fact, the attempt made by Eve to learn the inaccessible information would cause disturbance in an incompatible observable [28], which is maximal for mutually unbiased bases [29].

In contrast, the sender uses $\frac{n}{2}$ copies of a Bell state (or equivalently an adequate number of mutliqubit entangled state) and inserts them randomly in the string of travel qubits in the GV subroutine. This causes geographical separation between the entangled qubits (ensured here through application of permutation of particles (PoP)) and Eve is unaware of the particle pairs, thus may tend to choose wrong particle pairs to perform Bell measurement leading to entanglement swapping. Thus, at the receiver's end when correct positions of the entangled states are available, he can detect the signatures of eavesdropping in the form of entanglement swapping caused due to it [13,30]. An appropriate use of PoP technique makes the measurement basis unavailable to Eve and security comes from the orthogonal states only. The name GV subroutine originates from an orthogonal state based QKD scheme introduced by Goldenberg and Vaidman, where temporal separation between two localized wavepackets (whose orthogonal superpositions were used to send 0 and 1 ) were used to achieve the security [5].

Interestingly, the set of all entangled state based protocols which use BB84 subroutine can be easily transformed to corresponding completely orthogonal state based schemes by replacing the BB84 subroutine with GV subroutine (see [13, 18] for detail).

Also note that all the protocols described in this section and the next section assume that the buyer (Alice) and merchant (Bob) are registered members of eBay (eBay is an e-commerce corporation, but it can be equivalently viewed as an online portal or a bank, too), and eBay (Charlie) is capable to authenticate the identities of Alice and Bob while communicating with them. Further, in all the protocols described below, Alice sends a classical information $M$ via quantum means to Bob. Here, $M$ is the shopping information of Alice which includes her customer id, items to be purchased (item numbers), quantity of each item to be ordered, etc.

To begin with let us briefly describe Chou et al.'s protocol [23], which we refer here as CLZ protocol.

\subsection{Chou, Lin, Zeng (CLZ) protocol}

CLZ 1: Alice informs Charlie that she wants to purchase something online. After receiving this information, Charlie prepares and sends her a sequence of $2 n$ qubits that is randomly prepared in $\{|0\rangle,|1\rangle,|+\rangle,|-\rangle\}$. However, Charlie does not disclose which qubit is prepared in which basis.

Out of these $2 n$ qubits, $n$ will be used as decoy/verification qubits which will be used for eavesdropping check. 
In the original CLZ protocol, Charlie used to send $n+\delta$ qubits, out of which $\delta$ were verification qubits, but for unconditional security we need to check half of the received qubits]. This is why we use $\delta=n$ in this paper.

CLZ 2: Alice randomly selects $n$ of the $2 n$ qubits received by her and in collaboration with Charlie applies BB84 subroutine on those $n$ qubits. If the computed error rate is found to be lesser than the tolerable limit they continue to the next step, otherwise they restart the protocol.

After the eavesdropping check is performed using BB84 subroutine, the qubits used for the same are discarded, and Alice is left with $n$ qubits which she uses as message qubits in the next step.

CLZ 3: Alice encodes her shopping information $(M)$ on the $n$ qubits of her possession using following rule: to encode 0 (1) she does nothing (applies $i Y$ operator). Subsequently, she randomly inserts $n$ decoy qubits prepared at random in $\{|0\rangle,|1\rangle,|+\rangle,|-\rangle\}$ into the message encoded sequence and sends that to Bob.

The encoding operation here is the same as that used in LM05 protocol [8] of QSDC.

CLZ 4: After receiving an authenticated acknowledgment of the receipt of $2 n$ qubits from Bob, Alice discloses the positions of $n$ decoy qubits, and Alice and Bob apply BB84 subroutine on the decoy qubits to check eavesdropping. If no eavesdropping is found they go to the next step, otherwise they restart the protocol.

CLZ 5: Bob asks Charlie, for the initial states of the $n$ message qubits available with him, and Charlie provides that information. With the encoded qubits and their initial states, merchant Bob can now deduce the shopping information of the customer.

\subsection{Huang, Yang, Jia (HYJ) protocol}

Huang et al. [24] showed that in CLZ 3, when Alice sends a message encoded sequence to Bob, Charlie can capture all the qubits and replace them by a fake sequence of $2 n$ qubits. Later, when in CLZ 4, Alice discloses the positions of decoy qubits, Charlie discards them from the captured sequence and measures rest using the basis in which he had initially prepared the qubits. Eavesdropping check will definitely reveal this, but by then Charlie will have all the information encoded by Alice although this information was not intended for him. For instance, assume that a country wishes to buy some items for defense (say, weapons) from a multinational company, which would prefer to keep the details of the deal secret as its policy, and the buyer would not like to reveal this information (specifically, which items he is going to buy and the quantities of each item to be purchased) to a third party helping in making the payments. This limitation of CLZ protocol was circumvented in the HYJ protocol, which may be viewed as an improved version of the CLZ protocol. HYJ protocol may be described as follows:

HYJ 1: Same as CLZ 1.

HYJ 2: Same as CLZ 2.

HYJ 3: Same as in CLZ 3 with a difference that Alice also prepares a random key $K$, and instead of $M$ she sends $M^{\prime}=$ $K \oplus M$ to Bob and keeps $K$ secret.

HYJ 4: Same as CLZ 4.

HYJ 5(a): Alice announces $K$, and Bob uses that to obtain $M=K \oplus M^{\prime}$.

HYJ 5(b): Same as CLZ 5.

As $K$ is unknown to Charlie till eavesdropping is checked in HYJ 4, Charlie's strategy of sending fake sequence of qubits to Bob will not work here. Specifically, Charlie's eavesdropping will be detected in HYJ 4, and $K$ is announced in HYJ 5 if and only if no trace of eavesdropping is detected in HYJ 4. Thus, HYJ scheme is free from the limitation of the CLZ protocol. However, there exists a limitation of HYJ protocol as we have already mentioned. As $K$ is not known to Bob, Alice has freedom to change his order till HYJ 5(a) by disclosing a different key $K^{\prime}$. Let us explain this with a specific example. Consider that if Alice wants to buy a TV she has to send $M_{1}=100101$; and if she wants to buy a refrigerator then she has to send $M_{2}=111100$. In HYJ 3, Alice uses $K=010010$ as key to yield $M^{\prime}=K \oplus M_{1}=010010 \oplus 100101=110111$, but at the time of disclosure of the key she changes her mind and announces $K^{\prime}=001011$ as her key. As a consequence, Bob will decode the message as $K^{\prime} \oplus M^{\prime}=001011 \oplus 110111=111100=M_{2}$. It is possible to cease this freedom of Alice to change order till the end. However, to do so we have to ensure that Alice is not able to change the key after HYJ 3. It is possible if a copy of the key is already available with Bob. Thus, Alice and Bob need to implement a protocol of QKD,

\footnotetext{
${ }^{1}$ In Ref. [31], it is shown that a random test of half of the qubits provide an upper bound on the errors in the rest of the transmitted qubits.
} 
quantum key agreement, direct secure quantum communication first to create or distribute a key and subsequently use that key for encryption. This would restrict Alice from changing her order at the last moment.

One may argue that this freedom to choose the merchandise for Alice cannot affect the task intended. However, suppose that possibility of placing an order is allowed only for the goods available in the store. In that case, Alice may change her order to buy anything that may be available later. Further, on numerous such occasions (as in online limited offer sale, which start on a predecided time and the orders made before and after the start of the sale must be treated independently), this freedom of Alice is not desired. In what follows, we describe a few new schemes for quantum online shopping which are free from the above mentioned limitation of HYJ protocol.

\section{New protocols for quantum online shopping}

In this section, we report four different protocols for quantum online shopping. All of them are essentially modified schemes for CDSQC.

\subsection{Protocol 1: PoP based quantum online shopping using single photons}

Firstly, we show a simple minded PoP based scheme which is equivalent to HYJ protocol. The protocol is described as follows (remaining steps are the same as that in HYJ protocol).

PoP 3: Same as in CLZ 3 with a difference that Alice applies a permutation operator $\Pi_{n}$ on her message encoded sequence before random insertion of the decoy qubits, but keeps the actual sequence secret.

PoP 5(a): Alice announces $\Pi_{n}$ and Bob uses that to obtain $M$.

\subsection{Protocol 2: Orthogonal state based quantum online shopping using Bell states}

Our orthogonal state based quantum online shopping protocol can be described in following steps:

OSB 1: Charlie prepares $n$ Bell states $\left|\psi^{+}\right\rangle^{\otimes n}$ with $n \geq 2$, where $\left|\psi^{ \pm}\right\rangle=\frac{|00\rangle \pm|11\rangle}{\sqrt{2}}$ and $\left|\phi^{ \pm}\right\rangle=\frac{|01\rangle \pm|10\rangle}{\sqrt{2}}$. He prepares two ordered sequences from the Bell states as follows:

1. A sequence of all the first qubits of the Bell states: $P_{A}=\left[p_{1}\left(t_{A}\right), p_{2}\left(t_{A}\right), \ldots, p_{n}\left(t_{A}\right)\right]$,

2. A sequence of all the second qubits of the Bell states: $P_{B}=\left[p_{1}\left(t_{B}\right), p_{2}\left(t_{B}\right), \ldots, p_{n}\left(t_{B}\right)\right]$, where the subscripts $1,2, \cdots, n$ denote the order of a particle pair $p_{i}=\left\{t_{A}^{i}, t_{B}^{i}\right\}$, which is in the Bell state.

OSB 2: Charlie applies an $n$-qubit permutation operator $\Pi_{n}$ on $P_{B}$ to create a new sequence as $P_{B}^{\prime}=\Pi_{n} P_{B}$. Charlie withholds the information of the actual order $\left(\Pi_{n}\right)$ to restrict Bob from decoding Alice's message prior his permission to do so. Therefore, Bob need not bother about Alice's choice of merchandise before Charlie informs that payment has been made by her.

Without the knowledge of the permutation operator $\Pi_{n}$, a potential eavesdropper will also be ignorant about the particle pairs in the Alice's and Bob's strings $P_{A}$ and $P_{B}^{\prime}$, respectively.

OSB 3: Charlie subsequently prepares $2 n$ decoy qubits as $\left|\psi^{+}\right\rangle^{\otimes n}$ and randomly inserts the first (last) $n$ Bell states as decoy qubits in $P_{A}\left(P_{B}^{\prime}\right)$ to yield a larger sequence $P_{A}^{\prime \prime}\left(P_{B}^{\prime \prime}\right)$ having $2 n$ qubits.

It would be relevant to mention that the choice of Bell states as decoy qubits (i.e., GV subroutine) is not unique here. The same task can also be achieved using BB84 subroutine 2 or using other entangled states. As we are restricting ourselves to a completely orthogonal state based online shopping scheme we are discussing only random insertion of Bell pairs in the sequence of message qubits. Finally, Charlie sends $P_{A}^{\prime \prime}$ and $P_{B}^{\prime \prime}$ to Alice and Bob, respectively.

OSB 4: Charlie discloses the positions of the decoy qubits and partner pairs in Bell states after receiving the authenticated acknowledgment of the receipt of the qubits from Alice and Bob. Alice and Bob apply GV subroutine to check error rate and if the computed error rate is found lower than the tolerable error limit, they go to the next step. Otherwise, they return back to OSB1.

\footnotetext{
${ }^{2}$ The BB84 and GV subroutines are shown to be equivalent in the ideal conditions, while over noisy channels this equivalence does not hold anymore [32].
} 
OSB 5: Alice can now encode her shopping information $M$ by performing suitable Pauli operation. It is predecided that $I, X, i Y$, and $Z$ Pauli operations will be used to encode $00,01,10$, and 11, respectively. Subsequently, Alice concatenates $n$ decoy qubits (with a prior intention to use GV subroutine for eavesdropping checking) in the message encoded qubits. Finally, Alice sends the enlarged sequence $P_{A}^{\prime}$ to Bob after applying the permutation operator $\Pi_{2 n}^{\prime}$.

Though Bob has now access to both $P_{A}^{\prime}$ and $P_{B}^{\prime}$, he will not be able to find out which particle is entangled with which particle and decode Alice's message. Thus, he needs Charlie's (Alice's) disclosure of $\Pi_{n}\left(\Pi_{2 n}^{\prime}\right)$ before decoding the message. Alice's permutation operation provides security against the Charlie's aforementioned participant attack [24].

OSB 6: Alice discloses $\Pi_{n}^{\prime}$ corresponding to decoy qubits. Alice and Bob perform GV subroutine on the decoy qubits Alice has inserted. If the errors are below tolerable limit they proceed, otherwise they abort the protocol.

OSB 7: Charlie discloses $\Pi_{n}$ and Alice announces $\Pi_{n}^{\prime}$, which allow Bob to decode Alice's message.

OSB 8: Since the initial Bell states and exact sequence are known, Bob measures the initially entangled partner pairs in the Bell basis and using the outcomes of his measurement, he decodes the order information sent by Alice.

This quantum online shopping scheme is using the idea of quantum cryptographic switch discussed in Refs. [16, 17, 33].

\subsection{Protocol 3: Quantum online shopping using entanglement swapping}

We will now introduce an entanglement swapping based quantum online shopping scheme, inspired by the direct secure quantum communication [14] and CDSQC [17] protocols based on entanglement swapping where communication is performed without actually transmitting the message encoded qubits. Specifically, our entanglement swapping based scheme works as follows.

ESB 1: Charlie prepares $n$ copies of a three qubit GHZ-like entangled state

$$
|\psi\rangle=\frac{1}{\sqrt{2}}\left(\left|\psi_{1}\right\rangle_{12}|a\rangle_{3} \pm\left|\psi_{2}\right\rangle_{12}|b\rangle_{3}\right),
$$

where $\left|\psi_{i}\right\rangle$ s are the Bell states such that $\left|\psi_{1}\right\rangle \neq\left|\psi_{2}\right\rangle$, and the single qubit states $|a\rangle$ and $|b\rangle$ are orthogonal to each other, i.e., $\langle a \mid b\rangle=\delta_{a, b}$. This restriction ensures that qubit 3 remains appropriately entangled with the remaining 2 qubits.

For instance, without loss of generality, we can assume that Charlie prepares $|\psi\rangle=\frac{1}{\sqrt{2}}\left(\left|\psi^{+}\right\rangle_{12}|0\rangle_{3}+\left|\psi^{-}\right\rangle_{12}|1\rangle_{3}\right)$.

ESB 2: Charlie prepares three strings $P_{A 1}, P_{A 2}$, and $P_{B}$ of qubits 1, 2, and 3 of all $n$ GHZ-like entangled state, respectively. He further performs a permutation operator $\Pi_{n}$ on $P_{B}$ to obtain $P_{B}^{\prime}$. This permutation operator ensures Charlie's control over the communication between Alice and Bob.

ESB 3: Subsequently, Charlie inserts $n$ decoy qubits randomly in all three strings to obtain enlarged strings $P_{A 1}^{\prime}, P_{A 2}^{\prime}$, and $P_{B}^{\prime \prime}$. The choice of decoy qubits depends upon type of subroutine predecided by the legitimate parties to be performed for eavesdropping checking. Thereafter, he sends $P_{A 1}^{\prime}$ and $P_{A 2}^{\prime}\left(P_{B}^{\prime \prime}\right)$ to Alice (Bob).

ESB 4: Same as OSB 4 but the choice of eavesdropping checking subroutine is arbitrary.

ESB 5: Alice prepares $n$ copies of $\left|\psi^{+}\right\rangle_{A_{1} A_{2}}$ to encode her secret information of items to be purchased. Specifically, she applies a $Z$ gate on one of the qubits of the Bell state to encode 1 and does nothing to send 0 . Therefore, the composite state Alice and Bob hold is $\left|\psi^{\prime}\right\rangle=\frac{1}{\sqrt{2}}\left(\left|\psi^{ \pm}\right\rangle_{A_{1} A_{2}}\left|\psi^{+}\right\rangle_{12}|0\rangle_{3}+\left|\psi^{ \pm}\right\rangle_{A_{1} A_{2}}\left|\psi^{-}\right\rangle_{12}|1\rangle_{3}\right)$.

ESB 6: Alice measures qubits $A_{1}$ and 1 as well as $A_{2}$ and 2 in Bell basis, while Bob can measure his qubits in the computational basis. Subsequently, she announces her measurement outcomes, which should reveal her message to Bob.

To understand this point we can write the state before measurement as

$$
\begin{aligned}
\left|\psi^{\prime}\right\rangle_{m} & =\frac{1}{2 \sqrt{2}}\left(\left\{\left|\psi^{+}\right\rangle_{A_{1} 1}\left|\psi^{+}\right\rangle_{A_{2} 2}+\left|\psi^{-}\right\rangle_{A_{1} 1}\left|\psi^{-}\right\rangle_{A_{2} 2} \pm\left|\phi^{+}\right\rangle_{A_{1} 1}\left|\phi^{+}\right\rangle_{A_{2} 2} \pm\left|\phi^{-}\right\rangle_{A_{1} 1}\left|\phi^{-}\right\rangle_{A_{2} 2}\right\}|m\rangle_{3}\right. \\
& \left.+\left\{\left|\psi^{+}\right\rangle_{A_{1} 1}\left|\psi^{-}\right\rangle_{A_{2} 2}+\left|\psi^{-}\right\rangle_{A_{1} 1}\left|\psi^{+}\right\rangle_{A_{2} 2} \mp\left|\phi^{+}\right\rangle_{A_{1} 1}\left|\phi^{-}\right\rangle_{A_{2} 2} \mp\left|\phi^{-}\right\rangle_{A_{1} 1}\left|\phi^{+}\right\rangle_{A_{2} 2}\right\}|\bar{m}\rangle_{3}\right),
\end{aligned}
$$

where $m$ corresponds to the message bit encoded by Alice and the upper (lower) sign in the right-hand side of the equation corresponds to $m=0$ (1). Thus, if Alice announces both her measurements resulted in the same (different) Bell state(s) then Bob's measurement outcome will be same as (different from) the bit encoded by Alice, i.e., $m(\bar{m})$.

ESB 7: Same as OSB 7. 
Note that the Bell state prepared by Alice to encode her message was measured by her only in ESB 6 . Therefore, message encoded qubits actually do not travel through the channel accessible to Eve at all. This further reduces the number of times eavesdropping checking is to be performed as the rounds of transmission of qubits is reduced. In view of some of our recent results [34-37], which show that the performance of a quantum cryptographic scheme (in presence of noise) decays with an increase in the number of rounds quantum communication is involved, we can predict that this scheme would be relatively more robust against channel noise.

\subsection{Protocol 4: Quantum online shopping using dense coding}

The GHZ-like entangled state discussed in the previous protocol can also be used to send message in a secure manner without relying on entanglement swapping.

DCB 1: Same as ESB 1.

DCB 2: Charlie prepares three strings $P_{A}, P_{B}$, and $P_{C}$ of qubits 1,2, and 3 of all GHZ-like entangled states, respectively. Here, Charlie need not perform a permutation operator as he can also ensure his control over the communication by keeping the third qubit with himself.

DCB 3: Subsequently, Charlie inserts $n$ decoy qubits randomly in both strings $P_{A}$ and $P_{B}$ to obtain enlarged strings $P_{A}^{\prime}$ and $P_{B}^{\prime}$, respectively. The choice of decoy qubits depends upon type of subroutine predecided by the legitimate parties to be performed for eavesdropping checking. Thereafter, he sends $P_{A}^{\prime}$ and $P_{B}^{\prime}$ to Alice and Bob, respectively.

DCB 4: Same as ESB 4.

DCB 5: Same as OSB 5, but Bob requires Charlie's measurement results for the string $P_{C}$. Also the eavesdropping checking subroutine is arbitrary.

DCB 6: Same as OSB 6.

DCB 7: Same as OSB 7, while Charlie announces the result of measurement performed on the third qubit in $\{|a\rangle,|b\rangle\}$ basis instead of the permutation operator.

DCB 8: Same as OSB 8.

So far we have discussed the possibility of performing quantum online shopping task with the help of single photons (in Protocol 1), Bell states (in Protocol 2), and three-qubit GHZ-like state (in Protocols 3 and 4). However, it is not limited to this small set of states. There exist several alternative approaches through which efficient online shopping schemes can be designed using a large class of states. Here, we briefly mention a generalized approach to all these alternative paths.

\subsection{Various alternative ways to perform quantum online shopping using multi-qubit entangled states}

The task in hand can be accomplished using other multi-qubit entangled states, too. Specifically, in a densecoding based direct communication scheme [15, 17, 36], Alice (Bob) possesses $p \leq \frac{N}{2}(N-p)$ qubits out of total $N$ qubits of a $N$-qubit entangled state (such as $W$ state, GHZ state, GHZ-like state, $Q_{4}$ state, $Q_{5}$ state, cluster state, $|\Omega\rangle$ state, Brown state). Alice encodes her message using a suitable set of unitary operations and sends the qubits to Bob, who measures all $N$ qubits in the basis they were prepared.

In the densecoding based online shopping scheme (analogous to Protocol 2), Charlie can randomly prepare one of the above mentioned multi-qubit states and sends $p$ qubit to Alice while $N-p$ qubits to Bob in a secure manner. He permutes Bob's qubits to maintain his control power. Thereafter, Alice and Bob can perform the task under Charlie's supervision.

Along the line of Protocol 3, a quantum online shopping scheme using entanglement swapping that can transmit an $s$-bit message can be designed using the quantum states of the form

$$
|\psi\rangle=\frac{1}{\sqrt{2^{s}}} \sum_{i=1}^{2^{s}}\left|e_{i}\right\rangle\left|f_{i}\right\rangle,
$$

where $\left|e_{i}\right\rangle$ is an N-qubit maximally entangled state (as $W$ state, GHZ state, GHZ-like state, $Q_{4}$ state, $Q_{5}$ state, cluster state, $|\Omega\rangle$ state, Brown state). Specifically, $\left\{\left|e_{i}\right\rangle\right\}$ is a basis set with maximally entangled basis vectors in $C^{2^{N}}: N \geq s$, while $\left\{\left|f_{i}\right\rangle\right\}$ is a basis set in $C^{2^{l}}: l \geq s \geq 1$ which may be separable. Thus, $|\psi\rangle$ (an $N+l$ qubit entangled state) is prepared 
by Charlie, who shares the string of first $N$ qubits with Alice and that of the last $l$ qubits with Bob in a secure manner. He had to perform a permutation operator on Bob string to maintain his control over shopping scheme. Thus, Alice can send her message by preparing extra $m$-qubit entangled state in $\left\{\left|e_{i}\right\rangle\right\}$ basis and measure her qubits in such a way that message is transmitted through entanglement swapping [14].

A quantum online shopping scheme without entanglement swapping can also be performed using quantum channel of the form in Eq. (3). Specifically, Charlie keeps the last $l$ qubits with himself and sends $p \leq \frac{N}{2}(N-p)$ qubits from the remaining qubits to Alice (Bob) in a secure manner. Using densecoding Alice encodes her message and sends $p$ qubits in a secure manner to Bob, who requires Charlie to inform him the measurement outcome of the $l$ qubits to decode Alice's message. Therefore, we have discussed a set of possible mechanism to obtain quantum online shopping schemes using a large set of quantum states.

Once Alice and Bob share an entangled state prepared by Charlie, she can teleport her choice of item to be purchased to Bob [9]. Analogous to all the schemes discussed so far he will need Charlie's assistance to reconstruct the details of Alice's order.

\subsection{Other tasks related to e-commerce}

With an escalated interest on quantum internet [38,39] the feasibility of implementation of quantum solutions for e-commerce, voting [40], sealed-bid auction [41], etc., has also enhanced. Therefore, here we list a set of tasks those can be performed using modified forms of the proposed schemes. So far we were considering the quantum online shopping where the merchant delivers the order to buyer once payment has been made and confirmed by his bank. We can consider nowadays with the advent of online availability of soft copies of books, magazines, audio, and video which would require a bidirectional communication. Specifically, a quantum bidirectional online shopping scheme based on controlled quantum dialogue [33,35] can be performed where Alice sends information of her order to Bob under the supervision of Charlie, while Bob sends some sample files to Alice. Depending upon the quality of sample file, Alice may choose to cancel her order for which her payment will be refunded. Protocols described here can be easily modified to design many schemes for secure online computation and communication. For example, we may think of online examinations (where a central body (Charlie) would authorize an examiner (Bob) either to evaluated the answer sheet of the candidate Alice or to send him the question paper/evaluated answer sheet), participation in webinars (where the convener (Charlie) would check whether the participant Alice is authorized (paid registration fees) to listen the talk of Bob, viewing of a particular channel in the TV set of a particular customer (where the service provider (Charlie) would check whether the customer Alice is authorized to see the Channel broadcasted by Bob. Multicontroller versions of all such schemes can also be designed [23]. We are not extending this list here, but numerous such situations exist where the current protocol or its simple variants will be useful.

\section{Security of the proposed protocols}

As the set of protocols proposed by us are variants of CDSQC schemes, in analogy of the CLZ [23] or HYJ [24] protocols, the security of the schemes can be established along the same line. However, the attack designed in Ref. [24] and loophole pointed out by us here are not applicable on the proposed schemes. In what follows, we can categorize the security against a set of attacks in the outsider's and participant's attacks.

\subsection{Security against outsider's attack}

Here, we will establish the security of the proposed schemes against Eve's individual attacks.

1. Intercept-and-resend attack: Eve may choose to replace the qubits sent by Charlie (or a sender in general) by freshly prepared qubits and send it to the receiver. Using this attack, Eve would succeed to get Alice's message if she intercepts the encoded qubits sent from Alice to Bob exploiting information of the initially prepared state. To circumvent this attack in Protocol 1, BB84 subroutine is performed for eavesdropping checking. Specifically, when Charlie and Alice (or Bob) compute error rates using the randomly inserted decoy qubits prepared in two mutually unbiased bases, and from which they can proceed with (discard) the protocol if the error rate is below (above) threshold. If Eve measures the intercepted qubits either in the computational or diagonal basis before sending the freshly prepared qubit in the measurement outcome, she would induce disturbance for the wrong choice of basis. Specifically, for $n$ travel qubits, eavesdropping checking is performed on $\frac{n}{2}$ decoy qubits. Suppose Eve measures $m$ qubits (which will have both decoy and message qubits). Without loss of generality, we can assume that an equal number of decoy and message qubits are measured, i.e., $\frac{m}{2}$ decoy qubits are measured out of the total number of $\frac{n}{2}$ decoy qubits. As the error rate is calculated using decoy qubits only, the fraction of the intercepted decoy qubits is $f=\frac{m / 2}{n / 2}=\frac{m}{n}$. Thus, the mutual information 
between the sender (Charlie) and Eve is $I(C: E)=f / 2$ as she (Eve) can choose the correct basis half of the time. In the rest half of the time, when she chooses the wrong basis, she would prepare states in the wrong basis resulting in the wrong measurement outcome at the receiver's end half of the time $e=\frac{f / 2}{2}=\frac{f}{4}$. Thus, $I(A: B)=\left(1-H\left[\frac{f}{4}\right]\right)$, where $H[u]$ is the Shannon binary entropy. A quantum cryptographic scheme works until $I(A: B) \geq I(A: E)$, which results in the present case as tolerable attack fraction $f \cong 0.68$ and corresponding error rate as $17 \%$ ( [36, 37] and references therein). Note that Eve's success probability for each attacked qubit is $\frac{3}{4}$, which would become very small for higher values of $m$ as it would approach $\left(\frac{3}{4}\right)^{m}$.

2. Entangle-and-measure attack: Instead of intercepting and measuring the transmitting qubit, Eve may choose to entangle her qubit (initially prepared in $\alpha|0\rangle+\beta|1\rangle$ ) with the travel qubits accessible to her. To obtain the secret she will measure her ancillae qubits at a later stage. However, during security checking, decoy states randomly prepared in $|0\rangle$, $|1\rangle,|+\rangle$, and $|-\rangle$ are measured in the computational and diagonal basis, which would result the wrong result with probability $|\beta|^{2}$ if she attacks $|0\rangle$ or $|1\rangle$ decoy state, while the states remain separable for the rest of the decoy qubits. Thus, total probability of detection of Eve in this attack is $\frac{|\beta|^{2}}{2}$ assuming all four decoy states are equally probable [36, 37].

3. Correlation-elicitation attack: In this attack, Eve may exploit availability of some of the qubits of the entangled quantum channel more than once to extract encoded message [42]. Specifically, Eve can check the parity of Bell states using two CNOT if both the qubits are accessible to her. In the proposed schemes, use of decoy qubits leaves signature of eavesdropping to be detected while checking subroutine.

4. Impersonation attack or Man-in-the-middle attack: Eve may try to play as the receiver (sender) to the sender (receiver). This attack can be prevented by using authentication of the identities of the sender and receiver before quantum communication [43,44]. The receiver should further inform the sender about the receipt of the transmitted qubits over such an authenticated classical channel.

5. Disturbance attack or modification attack: Eve may also attempt some denial of service attacks, where she does not intend to extract information but to misguide the legitimate parties only. She can disturb the content of the message by changing the order of qubits or applying random unitary operations on the encoded qubits. Note that this will not reveal any information to Eve but this attack will also be revealed during eavesdropping checking performed to ensure secure transmission of the qubits ( [36] and references therein).

A special case which requires attention is the orthogonal state based quantum online shopping scheme which is described above as Protocol 2. In Protocol 2, if Eve applies a single qubit operation (say $X$ gate) on all the transmitted qubits, it will not change the decoy states being two qubit Bell states, while the order information will be changed completely. Applicability of this attack is not restricted to the Protocol 2 of e-commerce, it's in fact applicable to many schemes whose security is ensured by using the GV subroutine. This is not a serious attack as it does not reveal any information to Eve, but it has a serious impact on e-commerce as it can be used to change the order. Interestingly, it's possible to circumvent this attack. To do so, Alice can send some redundant qubits and compare their measurement outcomes with Bob.

6. Trojan-horse attack: Eve may design attacks based on the implementation of the scheme [45-47]. Though a quantum cryptographic scheme can not be proved secure against trojan-horse attack using principles of quantum mechanics, various technical measures have been discussed in the recent past to circumvent these attacks [45-47].

\subsection{Security against participant's attack}

A participant attack is more powerful in multiparty quantum communication scheme as a legitimate user has more access to the useful information than Eve. Therefore, here we analyze the attacking strategy of each participant.

1. Charlie can try to extract the choice of merchandise by Alice as pointed out in Ref. [24], where this attack has been circumvented by the use of an extra key by Alice. However, here we have discussed that this provide an advantage to Alice, which would not be present if Alice and Bob share the quantum key used by her using a quantum key distribution or agreement scheme ( [2, 4, 25] and references therein). Here, we have proposed that Alice applies a permutation operation on the encoded qubits to defend from this attack.

Charlie can also exploit the fact that he is authorized to prepare the state to be used as quantum channel. As the proposed schemes would remain secure until the controller prepares the desired state, Alice and Bob can randomly choose to measure a few copies of such states to check correlations.

2. Alice can try to cheat by changing the item ordered, without being detected, even after payment has been made. We have pointed the feasibility of this attack in the e-commerce schemes proposed in Refs. [23, 24]. However, the present 


\begin{tabular}{ccc}
\hline Name of the protocol & $\eta$ & $\eta_{q}$ \\
\hline CLZ [23] & $\frac{1}{4}$ & $\frac{1}{3}$ \\
HYJ [24] & $\frac{1}{5}$ & $\frac{1}{3}$ \\
Semiquantum online shopping Protocol 1 [25] & $\frac{1}{23}$ & $\frac{1}{21}$ \\
Semiquantum online shopping Protocol 2 [25] & $\frac{1}{18}$ & $\frac{1}{16}$ \\
Protocol 1 proposed here & $\frac{1}{5}$ & $\frac{1}{3}$ \\
Protocol 2 proposed here & $\frac{2}{7}$ & $\frac{2}{5}$ \\
Protocol 3 proposed here & $\frac{1}{8}$ & $\frac{1}{6}$ \\
Protocol 4 proposed here & $\frac{2}{9}$ & $\frac{1}{3}$
\end{tabular}

Table 1: Comparison of qubit efficiency of the existing and the proposed protocols.

schemes take care of any such attack by either using a key which is already shared between Alice and Bob or by application of a permutation operation by Alice. In the latter case, if Alice chooses to reveal wrong permutation operator, the information shared by Charlie regarding the initial state preparation to Bob will fail to extract order details. Thus, the online shopping task will be aborted and Alice would not gain any advantage.

3. As mentioned in Ref. [23], Bob can try to know the order placed by Alice before Charlie authorizes him to do so. However, Bob will not be able to decode information regarding Alice's order without Charlie's assistance as he will remain ignorant about the initial state chosen by the supervisor.

\section{Qubit efficiency of the protocols}

The efficiency of a secure quantum communication scheme can be analyzed using a quantitative measure [48] defined as

$$
\eta=\frac{c}{q+b},
$$

where $c$ is the number of classical bits transmitted using $q$ number of qubits. In addition, $b$ bits of classical communication is also required, which does not include that used for eavesdropping checking.

There is one more parameter, often discussed to analyze the performance of a cryptographic scheme, which quantifies number of bits transmitted per qubit in a scheme. Note that this definition does not take into account the classical communication required in accomplishing certain task. Thus, we can use another quantitative measure as

$$
\eta_{q}=\frac{c}{q} .
$$

The comparative study performed here (summarized in Table 1) establishes that the proposed protocols are much efficient compared to the existing schemes [23-25]. Specifically, the qubit efficiency of the improved HYJ protocol is less than that of CLZ protocol as an extra $n$ bit classical communication of key by Alice is required in the former protocol. Our Protocol 1, which uses the same amount of quantum and classical resources as HYJ scheme, is equally efficient to that. The rest of our protocols use entangled states. Specifically, Protocol 2 uses Bell states and densecoding thus becomes most efficient scheme. Note that Protocols 3 and 4 use GHZ-like states while difference in qubit efficiency comes from the reason that densecoding cannot be used in Protocol 3, and Protocol 4 requires $n$ bit classical communication. Thus, Protocol 4 is also more efficient than previously existing HYJ protocol. Protocol 3 may not appear more efficient but has an intrinsic advantage that message encoded qubits are never accessible to Eve. This feature is quite consistent with our earlier observation that requirement of quantum resources increases for sophisticated and complex quantum cryptographic tasks [25, 49]. In fact, one can clearly see that semiquantum online shopping schemes [25] have very small values of both qubit efficiency and bits transmitted per qubit used in comparison to the rest of the online scheme in Table 1

If the number of bits transmitted per qubit used is considered then our Protocols 1 and 4 are equally efficient as CLZ and HYJ. Protocol 2 (3) is most (least) efficient in the set of quantum online shopping schemes discussed here. We have not compared the qubit efficiency of the proposed schemes with more recent schemes [26, 27] due to use of quantum teleportation and quantum key distribution in them to ensure the security of the online banking. Also, the semiquantum schemes for any cryptographic task are also less efficient than corresponding fully quantum schemes [25]. 


\section{Conclusions}

With advent of quantum technologies and feasibility of quantum internet in the near future, various socio-economic problems can be addressed using quantum solutions. Along this line quantum schemes for voting, auction and online banking have been introduced in the recent past. As far as online shopping is concerned, an improvement in the recently proposed single photon based scheme [23] has been proposed, which restricts the bank from accessing the order placed by the buyer [24]. Here, we have pointed out a loophole in the improved scheme [24] that the buyer may change the merchandise even after payment for his order has been made.

We further propose solution to such a participant attack by using a previously shared key instead of random key by the buyer or a permutation operator. Here, we have given a set of such schemes using different quantum channels based on various CDSQC schemes. Specifically, a single photon based improved scheme (Protocol 1), a cryptographic switch based scheme using Bell state (Protocol 2), and two 3-qubit state based schemes using entanglement swapping (Protocol 3) and densecoding (Protocol 4) are proposed. The entangled state based schemes are further shown generally implementable using a large set of schemes. Thus, the proposed schemes provide numerous possible ways to experimentally implement the quantum online shopping scheme.

From the set of proposed quantum online shopping schemes the single photon based proposed by us is as efficient as previous single photon based scheme [24]. The entanglement based schemes are more efficient than the single photon based schemes if densecoding is exploited. However, the entanglement swapping based scheme (Protocol 3) proposed by us is least efficient as densecoding can not be exploited in this case. On the other hand, this less efficient scheme has an intrinsic advantage as message encoded qubits neither travel from Alice to Bob nor are accessible to Eve. We have established the security of all the proposed schemes from both individual attacks of an outsider and participant attacks. We have not discussed the effect of noise as the same on CDSQC has already been reported by us in case of non-Markovian channels, which can be reduced to the Markovian channel in the limiting case [35]. Further, a clear prescription for the study of the effect noise is provided in ( [33-35] and references therein) using which single qubit (entangled state) based quantum communication schemes are shown to perform better in the amplitude and phase damping (collective noise) channels [34]. Following the same strategy the effect of noise on the proposed protocols can be studied with ease, and we expect Protocol 1 to perform better than others over the amplitude and phase damping channels, while the other schemes may be preferred when the qubits are transmitted in the collective noise.

We hope that the proposed alternatives of the quantum online shopping schemes will provide experimentalists numerous possibilities to realize the task in the near future.

Acknowledgment: KT and AP thank Defense Research \& Development Organization (DRDO), India for the support provided through the project number ERIP/ER/1403163/M/01/1603. They also thank Chitra Shukla for her interest in this work and some useful criticism of the work.

\section{References}

[1] Shor, P. W.: Polynomial-time algorithms for prime factorization and discrete logarithms on a quantum computer. In Proc. 35th Annual Symp. on Foundations of Computer Science, (1994) Santa Fe, IEEE Computer Society Press (1994)

[2] Bennett, C. H., Brassard, G.: Quantum cryptography: public key distribution and coin tossing. In: Proceedings of the IEEE International Conference on Computers, Systems, and Signal Processing, Bangalore, India, pp. 175-179 (1984)

[3] Ekert, A.K.: Quantum cryptography based on Bell's theorem. Phys. Rev. Lett. 67, 661-663 (1991)

[4] Bennett, C.H.: Quantum cryptography using any two nonorthogonal states. Phys. Rev. Lett. 68, 3121-3124 (1992)

[5] Goldenberg, L., Vaidman, L.: Quantum cryptography based on orthogonal states. Phys. Rev. Lett. 75, 1239-1243 (1995)

[6] Long, G. L., Liu, X. S.: Theoretically efficient high-capacity quantum-key-distribution scheme. Phys. Rev. A 65, 032302 (2002)

[7] Bostrom, K., Felbinger, T.: Deterministic secure direct communication using entanglement. Phys. Rev. Lett. 89, 187902 (2002)

[8] Lucamarini, M., Mancini, S.: Secure deterministic communication without entanglement. Phys. Rev. Lett. 94, 140501 (2005)

[9] Yan, F. L., Zhang, X. Q.: A scheme for secure direct communication using EPR pairs and teleportation. Euro. Phys. J. B 41, 75-78 (2004) 
[10] Zhu, A.D., Xia, Y., Fan, Q.B., Zhang, S.: Secure direct communication based on secret transmitting order of particles. Phys. Rev. A 73, 022338 (2006)

[11] Banerjee, A., Pathak, A.: Maximally efficient protocols for direct secure quantum communication. Phys. Lett. A 376, 2944-2950 (2012)

[12] Deng, F.-G., Long, G. L., Liu, X.-S.: Two-step quantum direct communication protocol using the Einstein-PodolskyRosen pair block. Phys. Rev. A 68, 042317 (2003)

[13] Yadav, P., Srikanth, R., Pathak, A.: Two-step orthogonal-state-based protocol of quantum secure direct communication with the help of order-rearrangement technique, 13, 2731-2743 (2014)

[14] Shukla, C., Pathak, A.: Orthogonal-state-based deterministic secure quantum communication without actual transmission of the message qubits. Quantum Inf. Process. 13, 2099-2113 (2014)

[15] Shukla, C., Kothari, V., Banerjee, A., Pathak, A.: On the group-theoretic structure of a class of quantum dialogue protocols. Phys. Lett. A 377, 518 (2013)

[16] Srinatha, N., Omkar, S., Srikanth, R., Banerjee, S., Pathak, A.: The Quantum Cryptographic Switch, Quantum Inf. Process. 13, 59-70 (2014)

[17] Pathak, A.: Efficient protocols for unidirectional and bidirectional controlled deterministic secure quantum communication: Different alternative approaches. Quantum Inf. Process. 14, 2195 (2015)

[18] Pathak, A.: Elements of Quantum Computation and Quantum Communication. CRC Press, Boca Raton, USA (2013)

[19] Wen, X.J.: An E-payment system based on quantum group signature. Phys. Scr. 82, 065403-065407 (2010)

[20] Wen, X.J., Nie, Z.: An E-payment system based on quantum blind and group signature. In: Proceedings of International Symposium on Data, Privacy, and E-Commerce, America (2010)

[21] Wen, X.J., Chen, Y.Z., Fang, J.B.: An inter-bank E-payment protocol based on quantum proxy blind signature. Quantum Inf. Process. 12, 549-558 (2013)

[22] Cai, X.Q., Wei, C.Y.: Cryptanalysis of an inter-bank E-payment protocol based on quantum proxy blind signature. Quantum Inf. Process. 12, 1651-1657 (2013)

[23] Chou, Y.-H., Lin, F.-J., Zeng, G.-J.: An efficient novel online shopping mechanism based on quantum communication. Electronic Commerce Research 14, 349-367 (2014)

[24] Huang, W., Yang, Y.-H., Jia, H.-Y.: Cryptanalysis and improvement of a quantum communication-based online shopping mechanism. Quantum Inf. Process. 14, 2211-2225 (2015)

[25] Shukla, C., Thapliyal, K., Pathak, A.: Semi-quantum communication: protocols for key agreement, controlled secure direct communication and dialogue, Quantum Inf. Process. 16, 295 (2017)

[26] Shao, A.-X., Zhang, J.-Z., Xie, S.-C.: An e-payment protocol based on quantum multi-proxy blind signature. Int. J. Theor. Phys. 56, 1241-1248 (2017)

[27] Zhang, J.-Z., Yang, Y.-Y., Xie, S.-C.: A third-party e-payment protocol based on quantum group blind signature. Int. J. Theor. Phys. 56, 2981-2989 (2017)

[28] Buscemi, F., Hall, M. J., Ozawa, M., Wilde, M. M.: Noise and disturbance in quantum measurements: an informationtheoretic approach. Phys. Rev. Lett. 112, 050401 (2014)

[29] Biham, E., Boyer, M., Boykin, P. O., Mor, T., Roychowdhury, V.: A proof of the security of quantum key distribution. Journal of Cryptology 19, 381-439 (2006)

[30] Shukla, C., Pathak, A., Srikanth, R.: Beyond the Goldenberg-Vaidman protocol: secure and efficient quantum communication using arbitrary, orthogonal, multi-particle quantum states. Int. J. Quantum Inf. 10, 1241009 (2012)

[31] Nielsen, M.A., Chuang I. L.: Quantum Computation and Quantum Informatiom. Cambridge University Press, New Delhi, 589 (2008) 
[32] Sharma, R. D., Thapliyal, K., Pathak, A., Pan, A. K., De, A.: Which verification qubits perform best for secure communication in noisy channel? Quantum Inf. Process. 15, 1703-1718 (2016)

[33] Thapliyal, K., Pathak, A.: Applications of quantum cryptographic switch: Various tasks related to controlled quantum communication can be performed using Bell states and permutation of particles. Quantum Inf. Process. 14, 2599 (2015)

[34] Sharma, V., Thapliyal, K., Pathak, A., Banerjee, S.: A comparative study of protocols for secure quantum communication under noisy environment: single-qubit-based protocols versus entangled-state-based protocols. Quantum. Inf. Process. 15, $4681(2016)$

[35] Thapliyal, K., Pathak, A., Banerjee, S.: Quantum cryptography over non-Markovian channels. Quantum Inf. Process. 16, 115 (2017)

[36] Banerjee, A., Shukla, C., Thapliyal, K., Pathak, A., Panigrahi, P. K.: Asymmetric quantum dialogue in noisy environment. Quantum Inf. Process. 16, 49 (2017)

[37] Banerjee, A., Thapliyal, K., Shukla, C., Pathak, A.: Quantum Conference, Quantum Inf. Process. 17, 161 (2018)

[38] Kimble, H. J.: The quantum internet. Nature 453, 1023-1030 (2008)

[39] Pirandola, S., Braunstein, S. L.: Physics: Unite to build a quantum Internet. Nature 532, 169-171 (2016)

[40] Thapliyal, K., Sharma, R. D., Pathak, A.: Protocols for quantum binary voting. Int. J. Quantum Info. 15, 1750007 (2017)

[41] Sharma, R. D., Thapliyal, K., Pathak, A.: Quantum sealed-bid auction using a modified scheme for multiparty circular quantum key agreement. Quantum Inf. Process. 16, 169 (2017)

[42] Song, J., Zhang, S.: Comment on: "Quantum exam". Phys. Lett. A 350, 174 (2006)

[43] Kanamori, Y., Yoo, S.M., Gregory, D.A., Sheldon, F.T.: On quantum authentication protocols. InGLOBECOM'05. IEEE Global Telecommunications Conference, IEEE. 3, 5 (2005)

[44] Ljunggren, D., Bourennane, M., Karlsson, A.: Authority-based user authentication in quantum key distribution. Phys. Rev. A. 62, 022305 (2000)

[45] Gisin, N., Ribordy, G., Tittel, W., Zbinden, H.: Quantum cryptography. Rev. Mod. Phys. 74, 145 (2002)

[46] Deng, F.-G., Li, X.-H., Zhou, H.-Y., Zhang, Z.-j.: Improving the security of multiparty quantum secret sharing against Trojan horse attack. Phys. Rev. A 72, 044302 (2005)

[47] Li, X.-H., Deng, F.-G., Zhou, H.-Y.: Improving the security of secure direct communication based on the secret transmitting order of particles. Phys. Rev. A 74, 054302 (2006)

[48] Cabello, A.: Quantum key distribution in the Holevo limit. Phys. Rev. Lett. 85, 5635-5638 (2000)

[49] Thapliyal, K., Sharma, R. D., Pathak, A.: Orthogonal-state-based and semi-quantum protocols for quantum private comparison in noisy environment. arXiv:1608.00101 v1 (2016) 